\title{
THE EFFECT OF THE NUMBER OF DEGREES OF KINEMATIC REDUNDANCY ON THE ACTUATION FORCES OF A PLANAR PARALLEL MANIPULATOR
}

\begin{tabular}{|r|l|}
\hline Journal: & Transactions of the Canadian Society for Mechanical Engineering \\
\hline Manuscript ID & TCSME-2017-0002.R1 \\
\hline Manuscript Type: & Article \\
\hline Date Submitted by the Author: & 15 -Sep-2017 \\
\hline $\begin{array}{r}\text { Complete List of Authors: } \\
\text { Is the invited manuscript for } \\
\text { consideration in a Special } \\
\text { Issue? : }\end{array}$ & $\begin{array}{l}\text { Lee, Aiden; Universite de Moncton, Mechanical Engineering } \\
\text { Coudreau, Roger; Universite de Moncton, }\end{array}$ \\
\hline Keywords: & $\begin{array}{l}\text { planar parallel manipulators, kinematic redundancy, actuation forces, } \\
\text { optimization }\end{array}$ \\
\hline
\end{tabular}




\title{
THE EFFECT OF THE NUMBER OF DEGREES OF KINEMATIC REDUNDANCY ON THE ACTUATION FORCES OF A PLANAR PARALLEL MANIPULATOR
}

\author{
Aiden Lee ${ }^{1}$, Roger Boudreau ${ }^{1}$ \\ ${ }^{I}$ Département de génie mécanique, Université de Moncton, Moncton, N.-B., Canada \\ E-mail: roger.a.boudreau@umoncton.ca
}

\begin{abstract}
This paper proposes a methodology consisting of a two-step optimization procedure for resolving the actuation forces of a kinematically-redundant planar parallel manipulator following a specified trajectory. Simulation results compare the performance of the manipulator when different degrees of kinematic redundancy are used. It is seen that the required forces are generally lower when there are more degrees of kinematic redundancy; however, more mechanical energy is required. It is also shown that in some cases, less degrees of kinematic redundancy can produce similar results from a force point of view, while requiring less energy. Furthermore, the importance of correctly choosing an initial configuration is presented. The proposed method finds an optimal initial configuration to start the optimization by considering a performance index along the entire trajectory. The evolution of the singularity loci as the manipulator moves along the trajectory demonstrates why certain initial configurations should not be used, even if they are optimal at the initial pose.
\end{abstract}

Keywords: planar parallel manipulators; kinematic redundancy; optimization; actuation forces. 


\section{INTRODUCTION}

Parallel manipulators are known to have larger payload-to-weight ratios than serial manipulators. However, their workspaces are smaller and usually contain many singular configurations in which they cannot sustain a wrench applied to the end effector. For planar manipulators with passive joints on each end of the links attached to the end-effector, these Type-2 singularities (Gosselin and Angeles 1990) occur when lines extending through the links attached to the platform meet at a point or are parallel to each other. Redundancy has been proposed to reduce or eliminate these singularities. Actuation redundancy (e.g. Merlet 1996, Nokleby et al. 2005, Zibil et al. 2007) consists of actuating a normally passive joint in one or more branches of the manipulator. Branch redundancy (e.g. Firmani et al. 2007, Zhao and Gao 2009, Wu et al. 2010) consists of adding an extra actuated branch or branches to a manipulator. In both cases, there exists an infinite number of solutions for the actuator torques. Kinematic redundancy consists of adding extra joints and links to the manipulator. In this case, there exists infinitely many solutions to the inverse displacement problem and thus to the inverse force problem.

The number of degrees of redundancy has an important effect on the performance of the manipulator. Wang and Gosselin (2004) added one degree of kinematic redundancy in a branch of a planar manipulator, a spherical manipulator, and a spatial manipulator. This significantly reduced the number of singular configurations when compared to the non-redundant manipulator. For the planar manipulator with a given orientation of the platform, the singularity locus was reduced from a quadratic curve for the non-redundant manipulator to only a point for the redundant manipulator. Mohamed and Gosselin (2005) used kinematic redundancy to reconfigure the platform of parallel manipulators. Ebrahimi et al. (2007) 
proposed and analysed a 3 -PRRR ${ }^{1}$ manipulator and showed that kinematic redundancy enlarges the workspace and, due to the infinite number of solutions to the inverse displacement problem, can eliminate singularities within it. Actuation schemes to follow a trajectory for a 3-RPRR manipulator based on the optimization of a geometrical measure of proximity to singular configurations and the condition number of the Jacobian matrices were also presented in Ebrahimi et al. (2008). Extra prismatic joints were added to a 3-RRR manipulator and an optimization algorithm based on the value of the determinant of the Type-2 Jacobian matrix was proposed to avoid singularities (Cha et al. 2007) and to determine allowable ranges for the prismatic joints (Cha et al. 2009) when following a trajectory. Kotlarsky et al. (2010) added one prismatic actuated joint in one branch of a 3-RRR manipulator. Various optimization strategies were used to avoid singularities and minimize pose errors.

This paper proposes a study of the effect of the number of degrees of kinematic redundancy (DOKR) on the performance of a manipulator. The proposed methodology is completely general. Different performance criteria could be chosen for optimization, and the method would be applicable to different redundant manipulator configurations.

The manipulator considered is the 3-PRPR (Ruggiu and Carretero 2009, Boudreau and Nokleby 2012). The first joint on each chain can be considered redundant. The reason for choosing this joint is explained later. Instead of actuating all three redundant joints, one or two can be actuated and the best fixed positions of the others can be determined by optimizing a performance criterion. The effect of using one, two or three DOKR is studied. Ruiz et al. (2015) studied the effect of the number of DOKR of a 3-PRRR manipulator using energy as the performance criterion to be minimized. Their results indicate that energy consumption decreases when the number of DOKR increases.

\footnotetext{
${ }^{1}$ The nomenclature is as follows. The first number indicates the number of branches, while $\mathrm{R}$ and $\mathrm{P}$ denote revolute and prismatic joints, respectively. Actuated joints are underlined. 
In the present study, the required actuation forces while the end-effector is subjected to a wrench as it follows a desired trajectory are computed and compared for manipulators with a different number of DOKR. It is assumed that the motion is slow, such as in a machining operation, and that dynamic effects are negligible. However, the velocities of each link are computed and constrained to be within specified limits.

The paper is organized as follows. The manipulators studied are first described. The static equations of the manipulator are then presented. The optimization procedure used is explained, followed by results and a conclusion.

\section{DESCRIPTION OF THE MANIPULATORS.}

In this study, a 3-RPR manipulator is compared to a 3-PRPR redundant manipulator. Each branch of the 3-R $\underline{P R}$ manipulator consists of a passive revolute joint attached to the base, followed by an actuated prismatic joint attached to the end effector by a passive revolute joint as shown in Fig. 1(a). The length of the actuator for branch $i$ is defined by $\rho_{i 2}$. A fixed reference frame is attached to the base centroid at point $\mathrm{O}$ and a mobile reference frame is attached to the end effector centroid at point $P$. The orientation angle $\varphi$ of the end effector is defined by the angle between the $X$ and $x$ axes.

The 3-PRRR shown in Fig. 1(b) is similar to the 3-RPR, except that it has an additional actuated prismatic joint (base prismatic joint) on each branch between points $O_{i}$ and $A_{i}$. They are fixed to the base and cannot rotate. All three of these actuators are aligned along the lines formed by the equilateral triangle generated from the base points $O_{i}$. The prismatic actuators between points $A_{i}$ and $B_{i}$ are henceforth called the distal prismatic joints. While $i$ denotes the branch number, $j$ denotes the placement of the actuator within the branch.

Either the first or the second prismatic joint could be chosen to be redundant. The optimization variable is the length of the redundant joint. Some problems might occur if the second joint in each chain were considered redundant. For a specified pose of the end-effector and a specified length of the second 
prismatic joint, multiple solutions for the length of the first link are possible. The multiple solutions may happen, since an arc whose radius equals the length of the second joint could intercept the axis of the first actuator twice (see for example, branches 2 and 3 in Fig. 1(b) where this case would happen). The first joint in each chain is therefore considered redundant and multiple solutions for the other (non-redundant) joint are avoided.

\section{KINEMATICS OF THE MANIPULATORS}

\subsection{Static forces}

The static force analysis was presented in Boudreau and Nokleby (2012) and is summarized here. Let $\mathbf{f}_{i}$ designate the axial force directed along the distal link of branch $i$ on the end effector, i.e., the force along actuator $i 2$. Let $\mathbf{n}_{i 2}$ represent a unit vector along the distal link from $A_{i}$ to $B_{i}$. The forces acting on the end-effector are thus $\mathbf{f}_{i}, i=1,2,3$. The actuation forces of the prismatic joints are equal to the axial forces, and the equilibrium equations on the end-effector (see Fig. 1(b)) lead to

$$
\left[\begin{array}{ccc}
\mathbf{n}_{12} & \mathbf{n}_{22} & \mathbf{n}_{32} \\
\mathbf{k}^{\mathrm{T}}\left(\mathbf{P} \mathbf{B}_{1} \times \mathbf{n}_{12}\right) & \mathbf{k}^{\mathrm{T}}\left(\mathbf{P} \mathbf{B}_{2} \times \mathbf{n}_{22}\right) & \mathbf{k}^{\mathrm{T}}\left(\mathbf{P} \mathbf{B}_{3} \times \mathbf{n}_{32}\right)
\end{array}\right]\left[\begin{array}{c}
\tau_{12} \\
\tau_{22} \\
\tau_{32}
\end{array}\right]=\left[\begin{array}{c}
\mathbf{f}_{\boldsymbol{e}} \\
m_{e z}
\end{array}\right]
$$

which can be rewritten as

$$
\mathbf{J}_{2}^{\mathrm{T}} \boldsymbol{\tau}_{2}=\mathrm{F}
$$

where $\tau_{i 2}$ is the actuation torque, i.e., the amplitude of $\mathbf{f}_{i}$ in the distal link of branch $i, \mathbf{P B} \mathbf{B}_{i}$ is the vector from $P$ to $B_{i}, \mathbf{k}$ is the unit vector in the $z$ direction, $\mathbf{f}_{e}$ is the resultant force applied by the end-effector, and $m_{e z}$ is the moment about point $\mathrm{P}$ in the $z$ direction applied by the end-effector on the environment. In Eq. (2), $\tau_{2}$ is the vector of actuation forces in the distal prismatic actuators, $\mathbf{F}$ represents the end-effector output force and moment, or wrench, and $\mathbf{J}_{2}^{T}$ is the transpose of the manipulator Jacobian matrix that relates these two quantities. If we eliminate the third element of $\mathbf{n}_{\mathrm{i} 2}$, which is zero for the planar case, $\mathbf{J}_{2}^{T}$ is a 3 x 3 matrix. 
Let $\tau_{i 1}$ represent the amplitude of the actuation force in the base link of branch $i$ and $\mathbf{n}_{i 1}$ the unit vector from $O_{i}$ to $A_{i}$. The actuation force vector in the base actuator $i 1$ is thus $\boldsymbol{\tau}_{i 1}=\tau_{i 1} \mathbf{n}_{i 1}$. The only other force acting along a base actuator axis is the component of the reaction to the distal actuation force. The actuation force in the base link of each branch can therefore be obtained by a simple projection of the actuation force in the distal link onto the base link.

$$
\tau_{i 1}=\tau_{i 2} \mathbf{n}_{i 2} \cdot \mathbf{n}_{i 1}
$$

In matrix form,

$$
\left[\begin{array}{l}
\tau_{11} \\
\tau_{21} \\
\tau_{31}
\end{array}\right]=\left[\begin{array}{ccc}
\mathbf{n}_{11} \cdot \mathbf{n}_{12} & 0 & 0 \\
0 & \mathbf{n}_{21} \cdot \mathbf{n}_{22} & 0 \\
0 & 0 & \mathbf{n}_{31} \cdot \mathbf{n}_{32}
\end{array}\right]\left[\begin{array}{l}
\tau_{12} \\
\tau_{22} \\
\tau_{32}
\end{array}\right] \Rightarrow \boldsymbol{\tau}_{1}=\mathbf{A} \boldsymbol{\tau}_{2}
$$

So, for a specified output wrench F, Eq. (2) can be used to compute the actuation forces in the distal prismatic joints, i.e.,

$$
\boldsymbol{\tau}_{2}=\mathbf{J}_{2}^{-\mathrm{T}} \mathrm{F}
$$

The actuation forces in the base prismatic joints can then be computed using Eq. (4).

\subsection{Singularities}

Singular configurations occur when the distal links are parallel or when the lines along these links meet at the same point. In the first case, the platform cannot sustain a force perpendicular to the links, while in the second case, an external moment cannot be sustained. Since there are infinitely many solutions to the inverse displacement problem, it is possible to choose a solution through optimization such that the configuration is not singular.

\section{OPTIMIZATION PROCEDURE}

In this work, the actuation forces required to sustain a wrench on the end-effector while following a trajectory are minimized. A point-to-point motion planning (PPMP) procedure wherein the optimized base actuator lengths at a point are used as the initial guesses for the following step is used. The lengths 
of the base prismatic joints $\left(\rho_{i 1}, i=1,2,3\right)$ were chosen as the optimization variables with upper and lower limits imposed on their stroke. A constraint on the velocity of all the prismatic joints (base and distal) was also imposed. The velocity and acceleration analysis is presented in Boudreau and Nokleby (2012).

\subsection{Effect of the Initial Configuration}

The initial configuration has a significant effect on the optimization results. In Boudreau and Nokleby (2012), the initial actuator lengths that were input in the minimization problem minimized the actuation forces at the initial pose of the trajectory. They were obtained using Particle Swarm Optimization (PSO).

In Boudreau and Nokleby (2012), and in the current research, the core optimization problem was written as

$$
\min _{\rho_{i 1}}\left[\begin{array}{l}
\boldsymbol{\tau}_{1} \\
\boldsymbol{\tau}_{2}
\end{array}\right]^{T}\left[\begin{array}{l}
\boldsymbol{\tau}_{1} \\
\boldsymbol{\tau}_{2}
\end{array}\right]
$$

subject to constraints on the stroke and the velocities of the actuators. At each step of the trajectory, the objective function was minimized and the optimized $\rho_{i 1}$ at step $k$ were used as the initial guess for step $k$ $+1$.

It was later found that the configuration that minimizes the forces at the initial pose does not necessarily provide the best overall results. To determine better initial conditions at the first point of the trajectory, a different procedure was used here. Instead of only minimizing the forces at the initial point, the entire trajectory was considered to determine the initial configuration. The MATLAB ${ }^{\circledR}$ function MultiStart using fmincon at 30 initial points was used with the following objective function on the entire trajectory. It was found that 30 initial guesses produced repeatable results with a very high likelihood, which presumably indicates that a global minimum was found.

$$
\min _{\rho_{i 1}} \max \left(\mathrm{U}_{k=1}^{k_{\text {end }}}\left|\tau_{i}^{k}\right|, i=1 \text { to } 6\right)
$$

In this equation, vector $\tau^{\mathrm{k}}$ is the vector of actuation forces comprised of $\tau_{1}$ and $\tau_{2}$ at step $k$. The $i^{\text {th }}$ element of vector $\tau^{\mathrm{k}}$ is $\tau_{i}^{k}$. The objective function thus represents the minimization of the maximum value 
of the absolute value of the actuation forces throughout the trajectory. The initial configuration corresponding to the case for which the maximum of the absolute value of all actuation forces was smallest was thus found and considered optimal. Using this initial configuration to start the same optimization as in Boudreau and Nokleby (2012) can produce better overall results. For the same spiral trajectory, the maximum force was reduced from $387 \mathrm{~N}$ to $315 \mathrm{~N}$. Either of the objective functions of Eq. (6) and (7) could have been used alone to solve this problem. However, in the present study, the objective function of Eq. (6) was used in conjunction with the one of Eq. (7) to obtain generally smaller actuation forces. It is expected that by using the two-step optimization procedure, less energy will be required.

\subsection{Variation of the Number of Degrees of Kinematic Redundancy}

The procedure used to perform the optimization with a different number of DOKR is provided in this section. When all the base link lengths are variable and optimized at every point of a trajectory, there are three DOKR. Two DOKR are obtained when the length of $\rho_{11}, \rho_{21}$ or $\rho_{31}$ is fixed, and one DOKR is obtained when either of the following link lengths are fixed: $\rho_{11}$ and $\rho_{21}, \rho_{11}$ and $\rho_{31}$, or $\rho_{21}$ and $\rho_{31}$. Finally, when all the base link lengths are fixed, the manipulator is non-redundant. In all cases, when some actuators are fixed, their lengths are obtained by optimization in the outer loop. Therefore, when all the base link lengths are fixed, the manipulator's performance is different from that of the 3-R $\underline{P R}$ described in Fig. 1, since the fixed positions of the base points $A_{i}$ are different. The optimization procedure is described in Fig. 2. The chart is specific for $A_{l}$ fixed, but it is generalizable. The following constraints were imposed.

$$
\begin{aligned}
& \rho_{i j_{\min }} \leq \rho_{i j} \leq \rho_{i j_{\max }} \\
& -\dot{\rho}_{i j_{\max }} \leq \dot{\rho}_{i j} \leq \dot{\rho}_{i j_{\max }}
\end{aligned}
$$

where $i=1,2,3$, and $j=1,2$. 


\section{RESULTS AND DISCUSSION}

\subsection{Effect of the Number of DOKR}

To determine the effect of the number of DOKR, a manipulator with $O_{i} O_{j}=0.3 \mathrm{~m}$ and $B_{i} B_{j}=0.05 \mathrm{~m}$ was chosen. A $100-\mathrm{N}$ force acts tangentially to the trajectory and opposite to the motion of the endeffector at point $P$ (see Fig. 1). A clockwise 10-Nm moment is also applied to the end-effector whose orientation is maintained constant at $\pi / 6 \mathrm{rad}$. The velocity of the end-effector is constant and equal to $0.005 \mathrm{~m} / \mathrm{s}$. The trajectory is the same logarithmic spiral as in Boudreau and Nokleby (2012). This curve was chosen since it is not symmetric, it covers a large portion of the workspace and it passes near singularity loci of the non-redundant manipulator. It is described by

$$
\rho=a e^{k \beta} \text { with } k=\cot \psi
$$

where $\rho$ is the spiral's radius for a given angle $\beta$, which varies from 0 to $2 \pi \operatorname{rad}$ for the trajectory shown in Fig. 3. Increments of $\pi / 400 \mathrm{rad}$ were chosen for $\beta$. The constant $a$ was set to $0.03 \mathrm{~m}$, and $\psi$ represents the angle between the tangent and the radial line from the origin of the spiral at $(-0.05,0) \mathrm{m}$ to the radial point $(\rho, \beta)$. Angle $\psi$ was chosen as $75^{\circ}$. The wrench applied by the manipulator in this case is thus

$$
\mathbf{F}=[100 \cos (\beta+\psi), 100 \sin (\beta+\psi) ; 10]^{\mathrm{T}} \mathrm{N} ; \mathrm{Nm}
$$

The stroke of the actuators (Eq. 8) was set between $0.01 \mathrm{~m}$ and $0.29 \mathrm{~m}$, while the velocities of the actuators were limited to $\pm 0.15 \mathrm{~m} / \mathrm{s}$. Figure 3 shows the trajectory and the constant orientation workspace of the 3-RPR manipulator shown in Fig. 1(a).

The optimization was run to determine the performance of the manipulator for all possible combinations of DOKR. The results are presented in Table 1. The energy required to follow the trajectory is the sum of the product of the actuation force and the displacement of each actuator at each step. When actuators are fixed, their optimal lengths remain the same as those indicated in Table 1. Figure 4 shows the joint torques, actuation scheme, joint velocities, and joint accelerations for the 3-DOKR manipulator, while Figs. 5 to 7 show the torques and actuation schemes corresponding to some 
manipulator configurations shown in Table 1 for different numbers of DOKR. The joint accelerations are not shown due to space limitations, but the maximum value for the cases shown in Table 1 was about 4 $\mathrm{m} / \mathrm{s}^{2}$

One could expect that the lowest maximum joint torque would occur when there are three DOKR. Table 1 shows that this is not necessarily true. One case with one DOKR (actuators 21 and 31 fixed) produces a lower value for the tested trajectory. In general, however, the results show that the maximum joint torques are smaller when more DOKR are used, but the opposite is true for the required energy. The results found here are in contradiction to the results of Ruiz et al. (2015). It should be noted, however, that forces were minimized here while energy was minimized in Ruiz et al. (2015) and forces were not reported. The computation of results for all eight manipulator configurations allows the user to decide which joints should be kept fixed, if any, during a given trajectory.

\subsection{Effect of the Initial Configuration}

As discussed in Section 4, the initial configuration was chosen by considering the maximum force on the entire trajectory instead of being based on joint torque minimization only at the initial pose. The initial configuration greatly influences the optimized results. In fact, it can cause the manipulator to be unable to avoid singular configurations later in the trajectory. This section shows why this can happen.

The situation described above can occur depending on the required trajectory and output wrench. For the sake of illustration, the same trajectory is used but the output force is opposite to that used to compute the results of Table 1. This modified wrench better illustrates why the manipulator may be forced to adopt singular configurations later in the trajectory even though forces are always being optimized.

The concept is more easily shown when one DOKR is used. Let us consider the case when the positions of actuators 21 and 31 are fixed at optimized positions. Simulations indicate that the optimized values are $\rho_{21}=0.139 \mathrm{~m}$ and $\rho_{31}=0.290 \mathrm{~m}$. Keeping these values constant, Fig. 8(a) shows a plot of the objective function $\left(\boldsymbol{\tau}^{T} \boldsymbol{\tau}\right)$ versus the variable length of actuator 11 at the first pose of the trajectory. If the 
initial value of $\rho_{11}$ were chosen based on the minimization of the objective function at this pose only, a length on the right-hand side (point B) would be chosen. The singular configuration is clearly indicated: the objective function tends towards infinity. As the end-effector moves along the desired trajectory, the values of $\rho_{11}$ that correspond to singular configurations change. In this case, the singular configuration moves to the right as the trajectory is followed. Near the $618^{\text {th }}$ step, the singularity locus reaches the right end of the graph, as shown in Fig. 8(b). Furthermore, it does so without ever leaving the graph intermediately. Therefore, it slowly restricts the optimal value of the design variable until the manipulator whose initial condition placed its optimized initial value at the rightmost end of the graph is forced to adopt a singular configuration for at least one step of the trajectory. This extreme case serves as an example to illustrate that a more suitable initial condition is the leftmost end of the admissible actuator lengths because choices on that side of the singularity locus never become restricted between it and the limiting lengths of the remaining design variable. When the minimization of the maximum force which occurred somewhere on the trajectory determines the initial configuration of the manipulator, point $\mathrm{A}$ is chosen as an initial value.

A similar situation can occur when there are two DOKR. Let us consider the case when the position of actuator 11 is fixed at an optimized position. Using the optimized value of $\rho_{11}=0.202 \mathrm{~m}$ as a constant value, objective function contours can be plotted as a function of $\rho_{21}$ and $\rho_{31}$. The singularity loci changes at each step along the specified trajectory. Figure $9(a-b)$ shows graphs at the initial point and at step 310 of the trajectory. Say the method of Boudreau and Nokleby (2012) were used. The PSO would have chosen an initial configuration such that both variable base actuator lengths took values placing the point $\left(\rho_{21}, \rho_{31}\right)$ in the upper right quadrant. Eventually, the movement of singularity loci would have restricted the possible optimal values as it did for the case of the 1-DOKR manipulators. In this case, the movement of the singularity loci would be such that forces tending towards singularity values would be found. However, when the entire trajectory is considered, the maximum force is limited to $358 \mathrm{~N}$. In short, 
knowledge of the behaviour of the objective function as the end-effector traces out the trajectory enabled the new optimization scheme to find an initial configuration that did not cause the manipulator to be forced into a singular configuration.

There may exist trajectories that would always force the manipulator towards a singular configuration when this PPMP strategy is used. This would happen if, for example, a 1-DOKR singularity locus similar to that shown in Fig. 8(a) were initially located at the left-hand side of the graph before reaching the right-hand side later in the trajectory, as in Fig. 8(b). Moreover, when three DOKR are used, the design variable restriction effect can also occur. However, it is probably less likely to happen because of the vastly increased number of non-singular configurations. It does not occur for the trajectory tested here.

\section{CONCLUSIONS}

This study presented the effect of using various DOKR on the performance of a kinematicallyredundant planar parallel manipulator. The performance criteria used in this study consisted of the actuator forces required when the end-effector is subjected to a wrench while following a prescribed trajectory. It was shown that the maximum forces required by the actuators generally decrease when the number of DOKR increases. However, the energy required generally increases when the number of DOKR increases. The results show that considering all the possible combinations of DOKR is beneficial since, in some cases, using less DOKR can provide a solution that requires lesser forces and less mechanical energy. Keeping certain base actuator lengths constant can enable the algorithm to find solutions requiring lesser forces because the objective function, which depends on the relative orientation of the distal links, changes significantly compared to when all DOKR are active. In certain cases, this yielded better results in terms of forces, but in almost all cases, energy expenditure was found to be inversely proportional to the number of DOKR. Although results are shown for only one trajectory, the proposed method was also applied to circular and linear trajectories. The results and conclusions were similar to those presented in this paper. 
A methodology proposing a two-step optimization procedure for resolving joint actuation forces when following a desired trajectory has been developed. Considering the entire trajectory to determine an optimal initial configuration improves upon the method proposed in Boudreau and Nokleby (2012) in which the initial configuration was chosen by optimization considering only the conditions at the first step of the trajectory. The algorithm was unaware of conditions to come further along the trajectory so it was vulnerable to the effect of the restriction of the design variables due to the singularity loci, as presented in Section 5. Using knowledge of the evolution of the objective function values over the desired trajectory as influenced by the initial conditions, the optimization algorithm proposed here overcame this limitation in cases where the one used in Boudreau and Nokleby (2012) could not. If the initial configuration were chosen based only on the initial pose, the manipulator could be forced to adopt singular configurations as it moves along the trajectory. The method presented considers that the task may be planned off line and that the initial configuration can be chosen freely.

In short, the method proposed in this article can reduce the chances of failure of a point-by-point motion planning algorithm and even improve its performance by simultaneously choosing an optimal initial configuration and fixing the right combination of base actuator lengths.

The proposed methodology is general and can be applied to any redundant manipulator. The objective function can be changed to optimize other performance criteria. While the results presented here pertain to a case study on a specific manipulator, it is expected that the conclusions drawn on the effect of the number of DOKR would apply to other configurations of redundant manipulators.

\section{ACKNOWLEDGEMENTS}

The authors would like to thank the Natural Sciences and Engineering Research Council of Canada for their financial support for this work. 


\section{REFERENCES}

Boudreau, R. and Nokleby, S. 2012. Force optimization of kinematically-redundant planar parallel manipulators following a desired trajectory. Mechanism and Machine Theory. 56(10): 138-155.

Cha, S.-H., Lasky, T. A. and Velinsky, S. A. 2007. Kinematically-redundant variations of the 3-RRR mechanism and local optimization-based singularity avoidance. Mechanics Based Design of Structures and Machines. 35: 15-38.

Cha, S.-H., Lasky, T. A. and Velinsky, S. A. 2009. Determination of the kinematically redundant active prismatic joint variable ranges of a planar parallel mechanism for singularity-free trajectories. Mechanism and Machine Theory. 44(5): 1032-1044.

Ebrahimi, I., Carretero, J. A. and Boudreau, R. 2007. 3-PRRR redundant planar parallel manipulator: inverse displacement, workspace and singularity analyses. Mechanism and Machine Theory. 42(8): $1007-1016$.

Ebrahimi, I., Carretero, J. A. and Boudreau, R. 2008. Kinematic analysis and path planning of a new kinematically redundant planar parallel manipulator. Robotica. 26(3): 405-413.

Firmani, F., Zibil, A., Nokleby, S.B. and Podhorodeski, R.P. 2007. Force-moment capabilities of revolute-jointed planar parallel manipulators with additional actuated branches. Transactions of the Canadian Society for Mechanical Engineering. 31(4): 469-481.

Gosselin, C. and Angeles, J. 1990. Singularity analysis of closed-loop kinematic chains. IEEE Transactions on Robotics and Automation. 6(3): 281-290.

Kotlarski, J., Thanh, T. D., Heimann, B. and Ortmaier, T. 2010. Optimization strategies for additional actuators of kinematically redundant parallel kinematic machines. In Proceedings of IEEE Conference on Robotics and Automation, Anchorage, Alas., May 3-8 2010. pp. 656-661.

Nokleby, S., Fisher, R., Podhorodeski, R. and Firmani, F. 2005. Force capabilities of redundantlyactuated parallel manipulators. Mechanism and Machine Theory. 40(5): 578-599. 
Merlet, J.-P. 1996. Redundant parallel manipulators. Journal of Laboratory Robotics and Automation. 8(1): 17-24.

Mohamed, M. G. and Gosselin, C. M. 2005. Design and analysis of kinematically redundant parallel manipulators with configurable platforms. IEEE Transactions on Robotics. 21(3): 277-287.

Ruggiu, M. and Carretero, J. A. 2009. Kinematic analysis of the 3-PRPR redundant planar parallel manipulator. In Proceedings of the 2009 CCToMM Symposium on Mechanisms, Machines, and Mechatronics, Québec, Qc, Canada, May 28-29 2009.

Ruiz, A. G., Fontes, J. V. C. and da Silva, M. M. 2015. The influence of kinematic redundancies in the energy efficiency of planar parallel manipulators. In Proceedings of the ASME 2015 International Mechanical Engineering Congress and Exposition, Houston, USA, Nov. 13-19 2015.

Wang, J. and Gosselin, C.M. 2004. Kinematic analysis and design of kinematically redundant parallel mechanisms. ASME Journal of Mechanical Design, 126(1): 109-118.

Wu, J., Wang, L. and You, Z. 2010. A new method for optimum design of parallel manipulator based on kinematics and dynamics. Nonlinear Dynamics. 61(4): 717-727.

Zhao, Y. and Gao, F. 2009. Dynamic formulation and performance evaluation of the redundant parallel manipulator. Robotics and Computer-Integrated Manufacturing. 25(4-5): 770-781.

Zibil, A., Firmani, F., S.B. Nokleby, and Podhorodeski, R.P. 2007. An explicit method for determining the force-moment capabilities of redundantly actuated planar parallel manipulators. ASME Journal of Mechanical Design. 129(10): 1046-1055. 
Table 1. Results produced by optimization for various DOKR

\begin{tabular}{|c|c|c|c|c|c|c|c|}
\hline \multirow{2}{*}{$\begin{array}{r}\text { Fixed actuator } \\
\text { Original result [16] }\end{array}$} & \multicolumn{3}{|c|}{$\begin{array}{c}\text { Optimized initial } \\
\text { configuration, } \\
\rho_{11}, \rho_{21} \text { and } \rho_{31}(\mathbf{m})\end{array}$} & \multirow{2}{*}{$\begin{array}{c}\begin{array}{c}\text { Maximum } \\
\text { force (N) }\end{array} \\
387\end{array}$} & \multirow{2}{*}{$\begin{array}{c}\begin{array}{c}\text { Max force } \\
\text { reduction* } \\
\text { (\%) }\end{array} \\
0.0\end{array}$} & \multirow{2}{*}{$\begin{array}{c}\begin{array}{c}\text { Required } \\
\text { energy } \\
(\mathbf{J})\end{array} \\
572\end{array}$} & \multirow{2}{*}{$\begin{array}{c}\begin{array}{c}\text { Energy } \\
\text { reduction* } \\
(\%)\end{array} \\
0.0\end{array}$} \\
\hline & 0.288 & 0.288 & 0.283 & & & & \\
\hline None & 0.054 & 0.202 & 0.290 & 315 & 18.6 & 583 & -1.9 \\
\hline 11 & 0.010 & 0.239 & 0.280 & 378 & 2.3 & 589 & -3.0 \\
\hline 21 & 0.259 & 0.290 & 0.017 & 360 & 7.0 & 311 & 45.6 \\
\hline 31 & 0.019 & 0.182 & 0.290 & 315 & 18.6 & 398 & 30.4 \\
\hline 11 and 21 & 0.290 & 0.290 & 0.290 & 387 & 0.0 & 324 & 43.4 \\
\hline 11 and 31 & 0.290 & 0.290 & 0.290 & 387 & 0.0 & 326 & 43.0 \\
\hline 21 and 31 & 0.074 & 0.118 & 0.255 & 298 & 23.0 & 345 & 39.7 \\
\hline 11,21 and 31 & 0.290 & 0.290 & 0.290 & 387 & 0.0 & 198 & 65.4 \\
\hline Non-redundant 3-RPR & \multicolumn{3}{|c|}{ Not applicable } & 666 & -72.1 & 345 & 39.7 \\
\hline
\end{tabular}

* Relative to the original result obtained with the method of Boudreau and Nokleby 2012 


\section{Figure Captions}

Fig. 1 (a) 3-R $\underline{P R}$ manipulator; (b) 3-PRRR manipulator

Fig. 2 Optimization procedure (example for link $\rho_{11}$ at a fixed length)

Fig. 3 Constant orientation $(\pi / 6)$ workspace of the $3-R \underline{P R}$ and spiral trajectory

Fig. 4 3-DOKR manipulator with optimized initial conditions

Fig. 5 Result obtained by fixing the length of actuator 31 and optimizing initial conditions

Fig. 6 Result obtained by fixing the length of actuators 21 and 31 and optimizing initial conditions

Fig. 7 Result obtained by fixing the length of all base actuators and optimizing initial conditions

Fig. 8 Evolution of the objective function, actuators 21 and 31 fixed and optimized (1-DOKR):

(a) initial pose; (b) step 618 of the 801-step trajectory

Fig. 9 Evolution of the objective function contours, actuator 11 fixed and optimized (2-DOKR):

(a) initial pose; (b) step 310 of the 801-step trajectory 
Transactions of the Canadian Society for Mechanical Engineering

Page 18 of 26
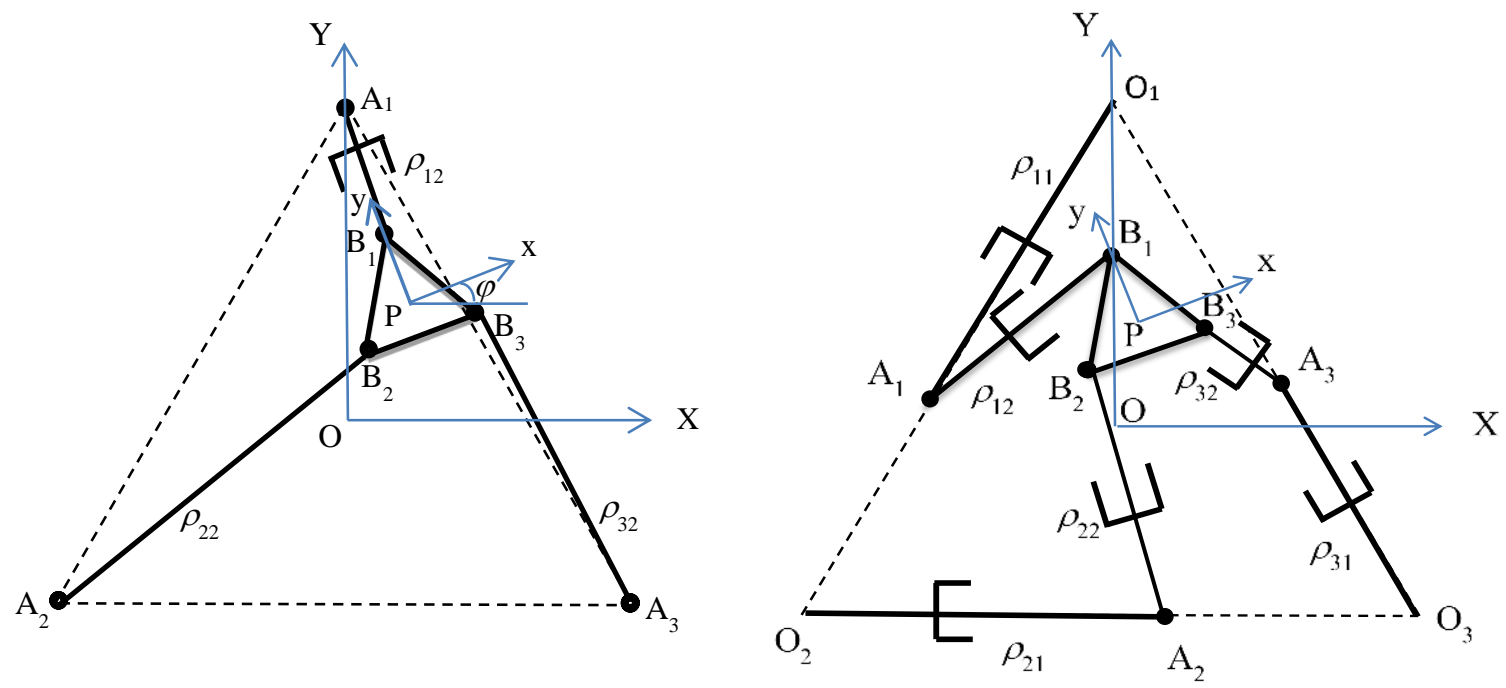

https://mc06.manuscriptcentral.com/tcsme-pubs 


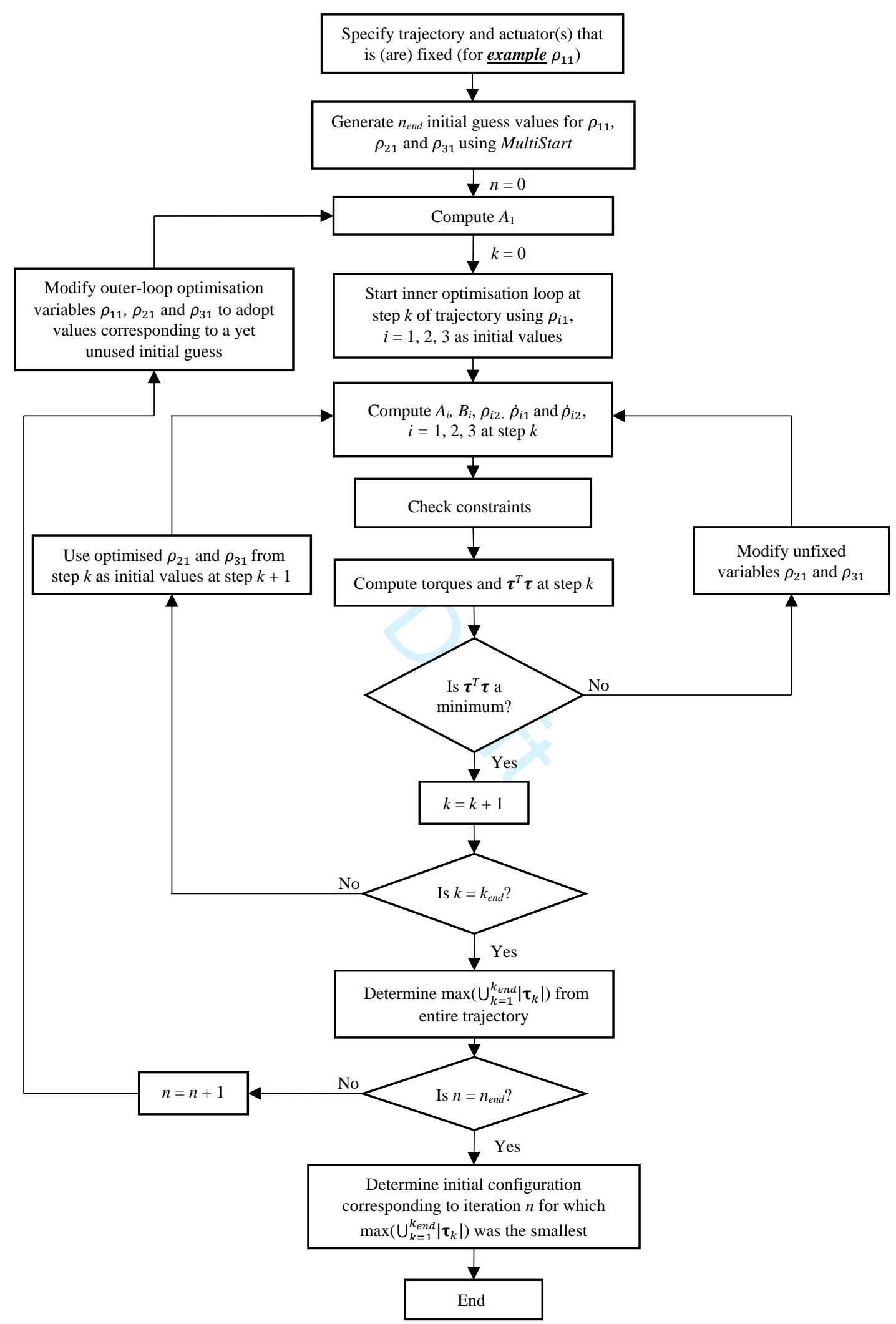




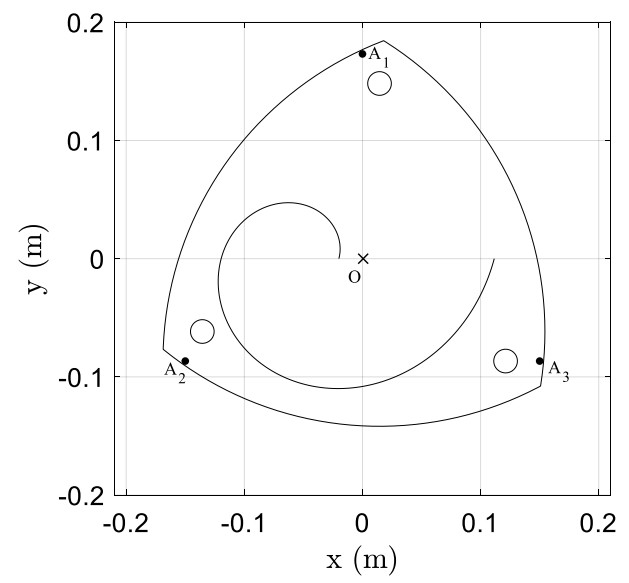

https://mc06.manuscriptcentral.com/tcsme-pubs 

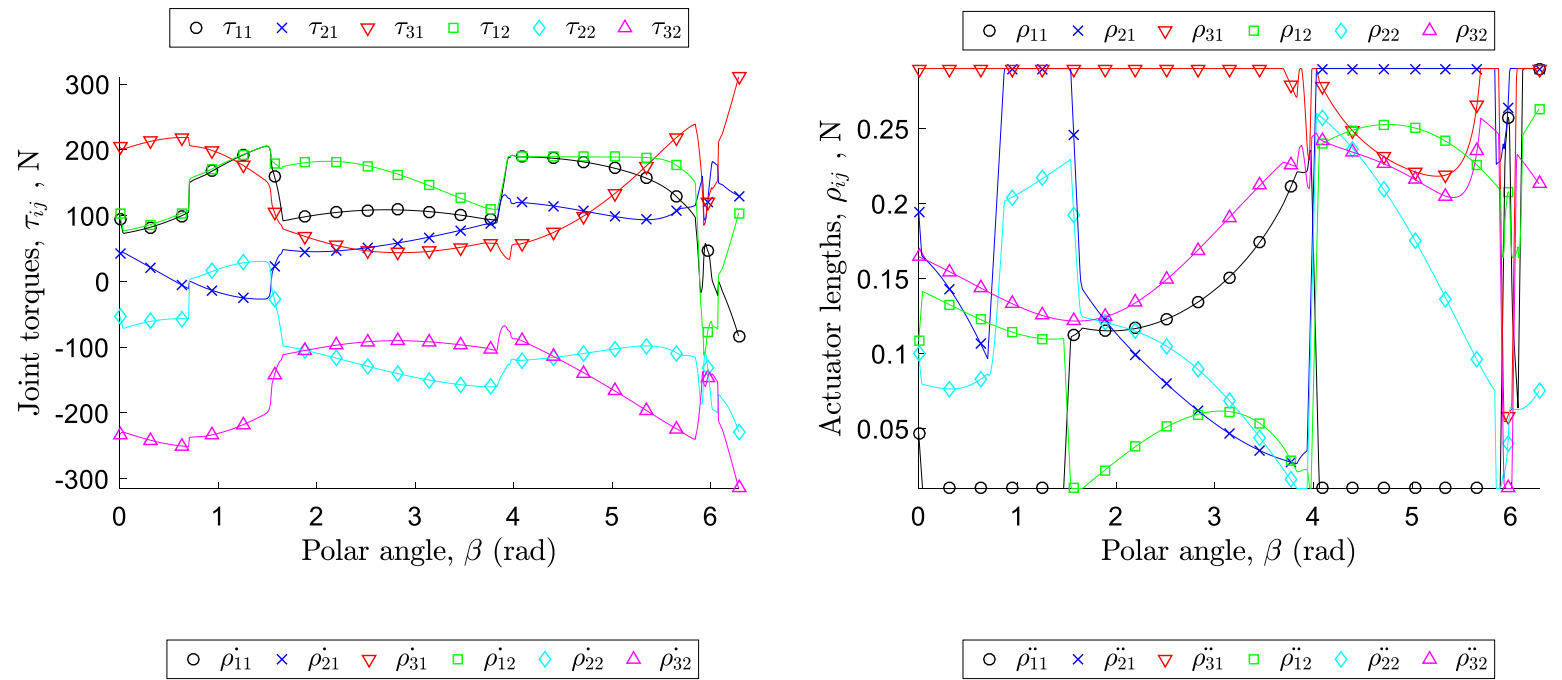

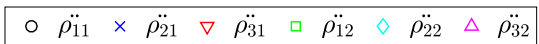
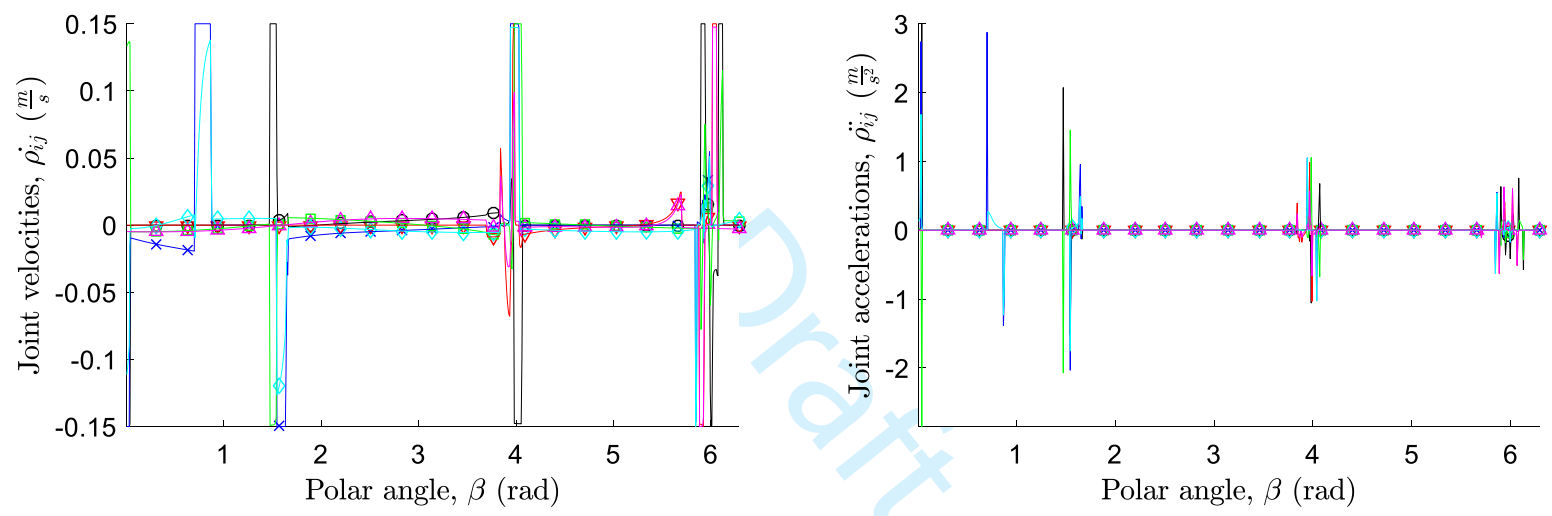

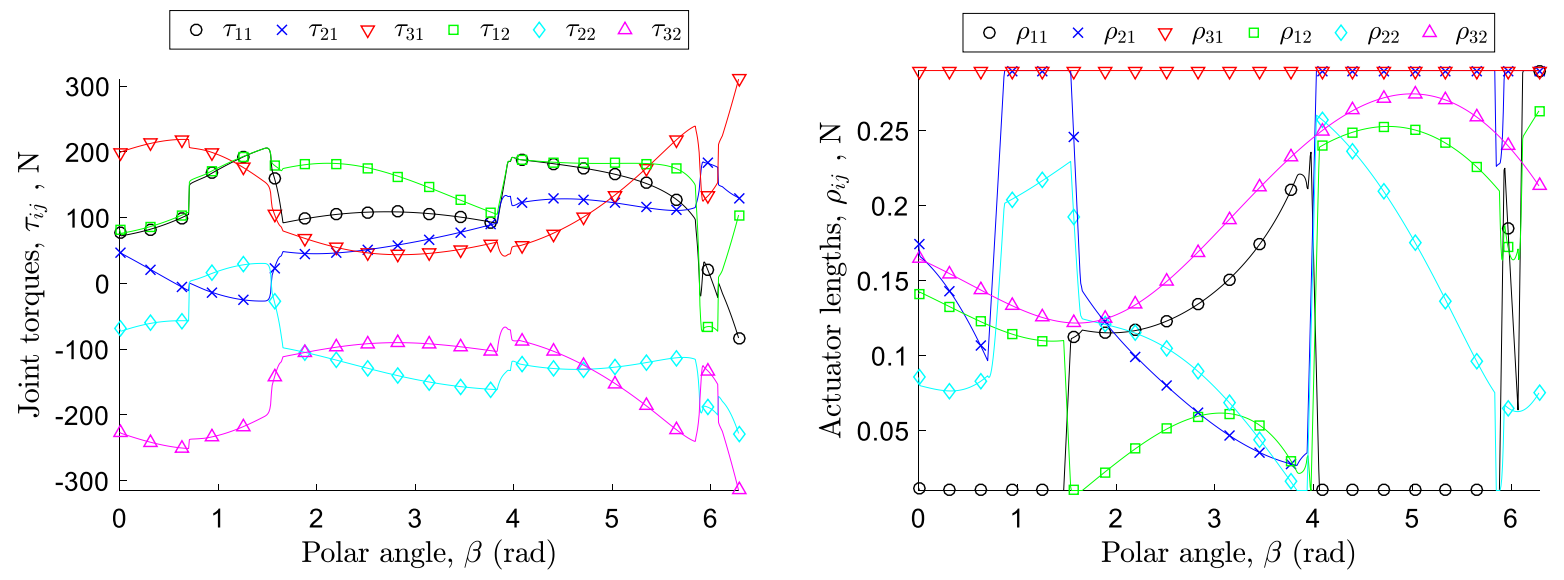

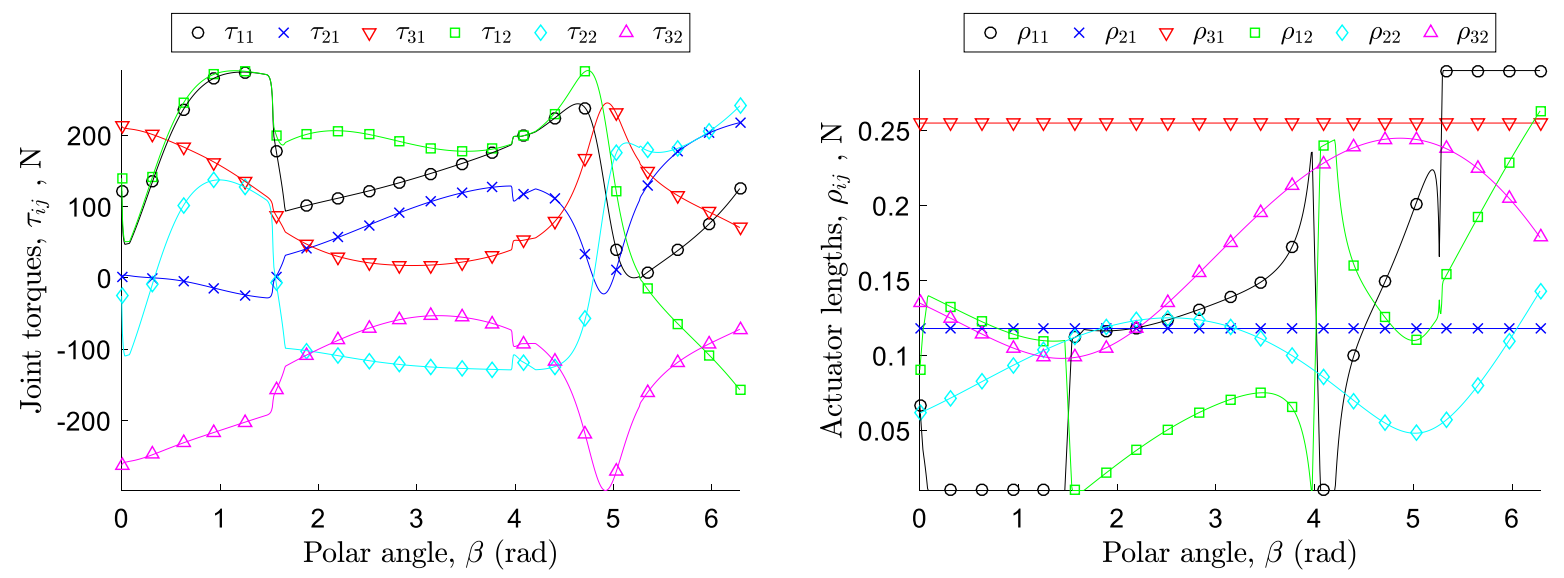

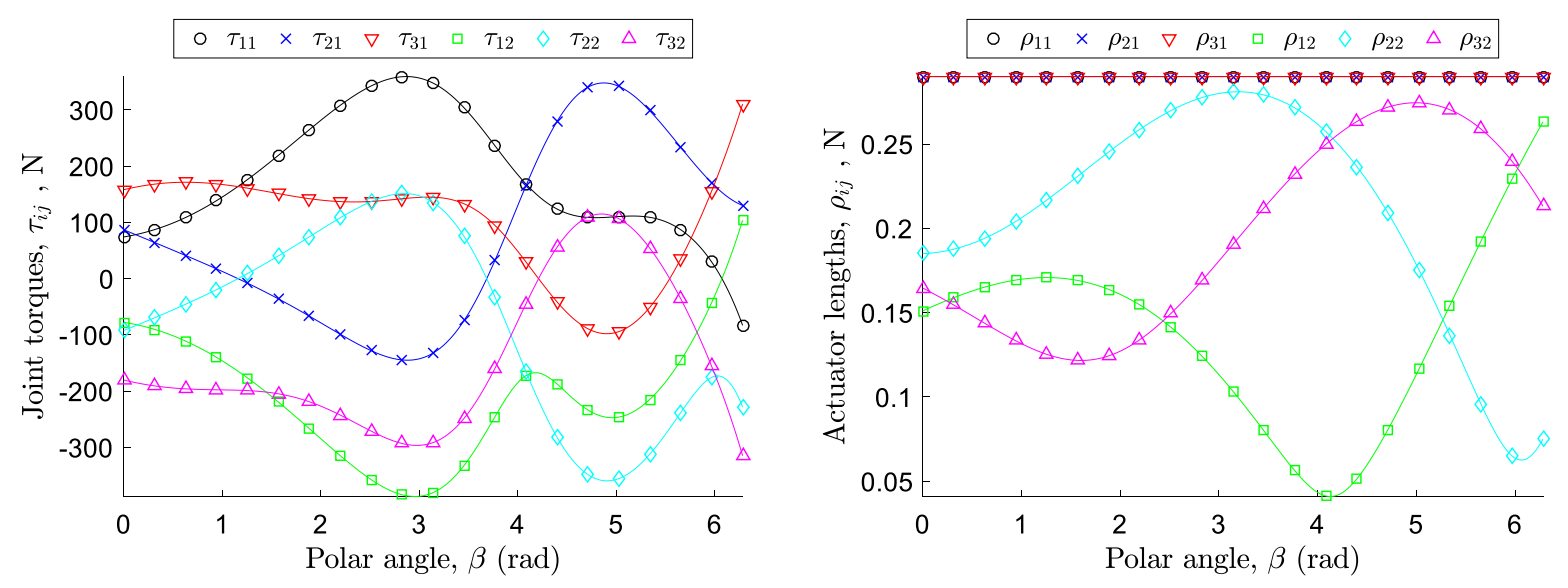


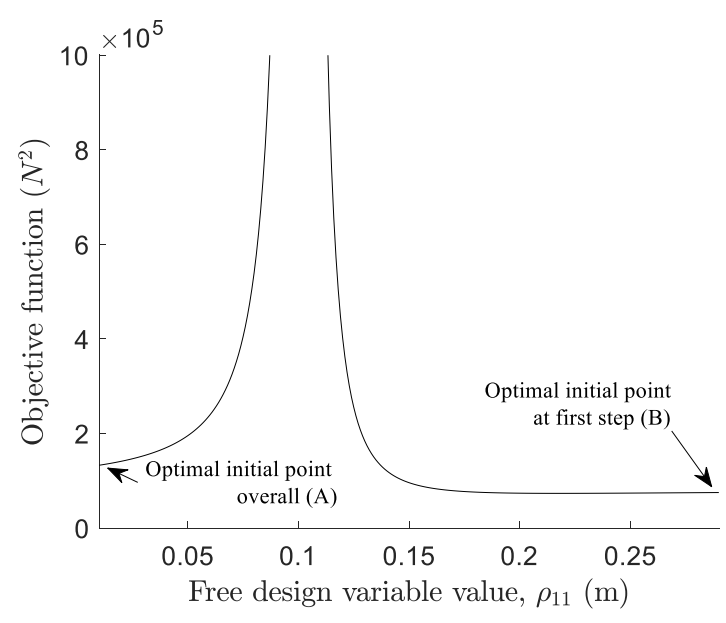

(a)

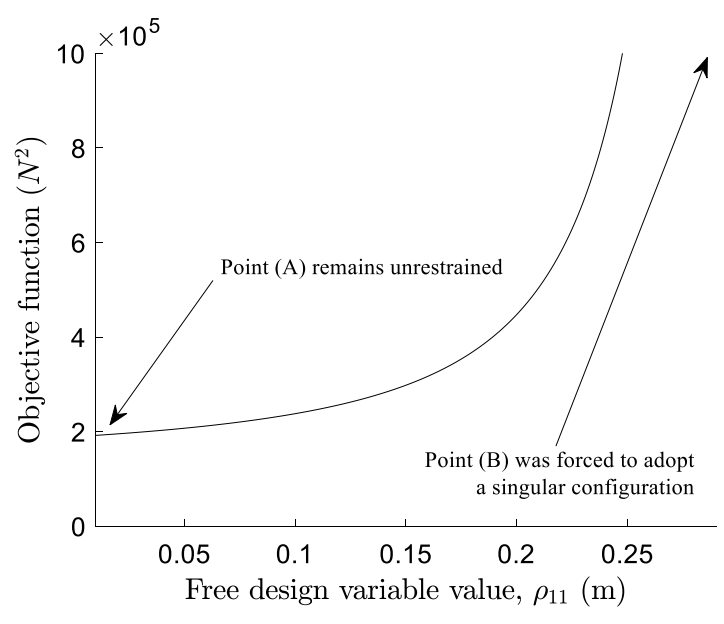

(b) 


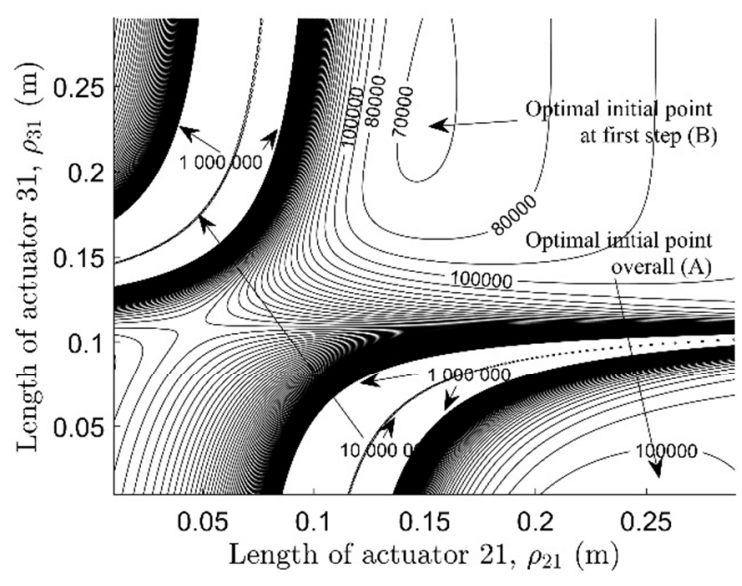

(a)

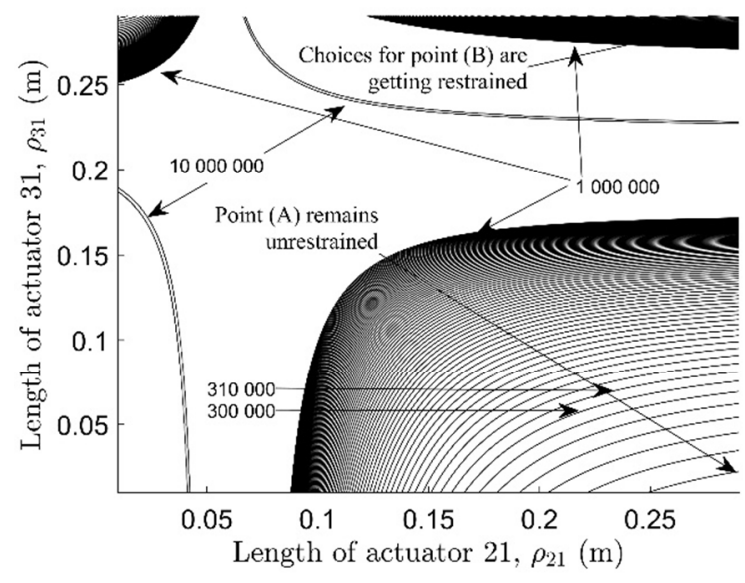

(b) 